\title{
Could Digital Therapeutics be a Game Changer in Psychiatry?
}

\author{
Chul-Hyun Cho ${ }^{1,2}$ and Heon-Jeong Lee, ${ }^{1,2,3 凶}$ \\ ${ }^{1}$ Department of Psychiatry, Korea University College of Medicine, Seoul, Republic of Korea \\ ${ }^{2}$ Chronobiology Institute, Korean University, Seoul, Republic of Korea \\ ${ }^{3}$ Department of Biomedical Science, Korean University College of Medicine, Seoul, Republic of Korea
}

Digital therapeutics (DT) is a new concept that encompasses therapeutic approaches that are used to change the patient's behavior using a variety of digital technologies and ultimately to treat diseases or promote health. ${ }^{1}$ DT has the potential to lead to natural and sustained behavioral changes similar to therapeutic interventions that have been confined to the clinic, through real-life approaches based on mobile or computer apps with various internet of medical things (IoMT). Furthermore, it is expected to change the paradigms of treatment. Psychiatric disorders and chronic diseases, in which behavioral change is key for good prognosis, are considered to be the most important applied targets of DT. ${ }^{2}$ In particular, it is actively applied to various psychiatric disorders such as substance abuse, attention-deficit/hyperactivity disorder, insomnia, panic attacks, anxiety, and depression, among others.

In the psychiatric field, biological therapy, which is represented by pharmacotherapy, and psychotherapy have been applied to clinical practice in two major ways. However, these conventional treatments have limitations in that they could only be performed face-to-face by therapists, or when compliance is poor, the effectiveness is reduced. In addition, psychiatric management in the stabilization (maintenance) phase, as well as the acute phase, is a very important part of psychiatric treatment. Furthermore, it is necessary to expand the therapeutic field with the concept of psychiatric rehabilitation. As such, the holes that still exist in the actual psychiatric fields are a task that needs to be densely filled to provide more complete psychiatric treatment. DT is expected to be able to play such an important role in achieving this task. ${ }^{3}$ Until now, most DTs have been implemented in the form of apps in the psychiatric field, and in recent years, DT

\footnotetext{
$\triangle$ Correspondence: Heon-Jeong Lee, MD, PhD

Department of Psychiatry, Korea University Hospital, Korea University College of Medicine, 73 Inchon-ro, Seongbuk-gu, Seoul 02841, Korea Tel: +82-2-9206721, Fax: +82-2-9297679, E-mail: leehjeong@korea.ac.kr

(a) This is an Open Access article distributed under the terms of the Creative Commons Attribution Non-Commercial License (https://creativecommons.org/licenses/bync/4.0) which permits unrestricted non-commercial use, distribution, and reproduction in any medium, provided the original work is properly cited.
}

is being defined as a broad area including virtual reality (VR). The US FDA approved the therapeutic application of DT in the psychiatric field promptly, such as for addiction, and thus promoting the quicker and more aggressive clinical application of DT.

What role can DT play in psychiatry? $?^{4}$ First, it can be considered to have a supplementary role with conventional treatment. DT will play a very important role in helping to perform psychotherapeutic sessions, such as cognitive-behavior therapy, in daily life, improving treatment compliance, selfmanaging the sleep-wake cycle, and modifying lifestyle habits, such as diet and exercise. In addition, the landscape of the clinic can be changed. The treatment will be based on the various IoMT data measured using digital devices and DT records, and DT prescriptions may be made alongside drug prescriptions by psychiatrists. On the other hand, DT may replace existing treatments partially or take on new roles. The application of the structured psychotherapeutic approach to DT will reduce the number of face-to-face treatments with therapists, which can be largely replaced by an Internet-based method. In addition, new therapies may emerge through the therapeutic application of new technologies such as VR.

Clinical applications of DT have already begun and are expected to become more common in the future. In particular, this could develop explosively because it is linked to an aspect of industry, and thus needs to be thoroughly prepared. What should mental health professionals such as psychiatrists prepare for in the DT era? First, we must respond to changes with openness and creativity. The development of new technologies and their application to health care will be accelerated. It is necessary to think about the application to psychiatry with openness and creativity on the premise of the development of digital technology. Experts who know the unmet needs in the clinical field will be able to proactively accept new technologies and play a role in implementing appropriate technologies to meet clinical needs. Second, we should approach the DT era from a critical perspective while maintaining professional conservatism. Since medicine is tar- 
geted at patients, it must be grounded in its efficacy and adverse effects through thorough and scientific validation such as randomized controlled trials. Clinical evidence is essential for this, and clinical research is the responsibility of the mental health professional. Third, research and development are needed by networking with experts from diverse fields based on convergent thinking. In the DT era, no-one can conduct in-depth and practical research alone. Only communication and collaboration among diverse experts as needed will be able to provide powerful research results. Fourth, we must maintain an integrated perspective with the clear goal of patient care. This means that the implementation of digital technology is not an end in itself, but that there is an unmet need for patient-centered care and that DT is to be implemented to fill that need. Therefore, there is a need to choose better treatments. For better therapeutic outcomes, various psychiatric treatments should be tightly integrated. ${ }^{5}$ Fifth, we have to be prepared to digest a huge amount of digital data. The vast amount of big data and digital phenotypes obtained through DT and IoMT will provide living information in the real world and will help establish new concepts of psychiatric disorders. ${ }^{6}$ It is necessary to think about well-formed digital research designs and research methodologies that go beyond evidence-based medicine. ${ }^{7,8}$ Sixth, we need to be literary and socialistic about new changes. This means that we must preemptively respond to issues surrounding medical ethics, psychiatrist-patient relationship, and patient data security in the DT era. ${ }^{9,10}$

Technology will advance, the treatment paradigm will change, and some will research and develop. We cannot predict the future accurately, but DT has already begun, and the dice have been thrown. DT will be a game changer by heralding a new treatment paradigm. The future will be for those who prepare. As the wind of change grows, the role of prepared mental health professionals will become more important. It is in this age that we need mental health professionals with conservative openness, networking based on convergent thinking and patient-centered integrative perspective.

\section{Acknowledgments}

This research was supported by the Brain Research Program through the National Research Foundation of Korea (NRF), funded by the Ministry of Science, ICT \& Future Planning (NRF-2016M3C7A1914448) and ICT \& Future Planning for convergent research in Development program for convergence R\&D over Science and Technology Liberal Arts (NRF- 2017M3C1B6070978).

\section{REFERENCES}

1. Natanson E. Digital therapeutics: the future of health care will be appbased. Forbes, 2017.

2. Kvedar JC, Fogel AL, Elenko E, Zohar D. Digital medicine's march on chronic disease. Nat Biotechnol 2016;34:239-246.

3. Baker JT, Germine LT, Ressler KJ, Rauch SL, Carlezon WA Jr. Digital devices and continuous telemetry: opportunities for aligning psychiatry and neuroscience. Neuropsychopharmacology 2018;43:2499-2503.

4. Bhui K. eHealth adventures in psychiatric therapeutics. Br J Psychiatry 2017;210:309-310.

5. Sverdlov O, van Dam J, Hannesdottir K, Thornton-Wells T. Digital therapeutics: an integral component of digital innovation in drug development. Clin Pharmacol Ther 2018;104:72-80.

6. Insel TR. Digital phenotyping: technology for a new science of behavior. JAMA 2017;318:1215-1216.

7. Chang S, Lee TH. Beyond evidence-based medicine. New Engl J Med 2018;379:1983-1985.

8. Topol EJ. High-performance medicine: the convergence of human and artificial intelligence. Nat Med 2019;25:44-56.

9. Parish MB, Fazio S, Chan S, Yellowlees PM. Managing psychiatrist-patient relationships in the digital age: a summary review of the impact of technology-enabled care on clinical processes and rapport. Curr Psychiatry Rep 2017;19:90.

10. Price WN 2nd, Cohen IG. Privacy in the age of medical big data. Nat Med 2019;25:37-43. 